\title{
„Meine eigene Zahnarztpraxis“ von Thomas Jans
}

So gut wie jeder Zahnmediziner stellt sich mindestens einmal in seinem Berufsleben die Frage: „Soll ich mich selbstständig machen oder bleibe ich angestellt?" In diesem Buch wird der Leser anhand von zwei Praxisbeispielen genau durch diesen Prozess geführt.

Martin Schönenstein // Witten

$\mathrm{V}$ on der grundsätzlichen Frage der Selbstständigkeit über die verschiedenen Praxisformen, die Kaufpreisermittlung, den Businessplan, das Bankgespräch sowie die Vertragsverhandlungen wird der Leser auf eine extrem kurzweilige Reise durch den Prozess des Starts in eine Selbstständigkeit mitgenommen.

Wer von diesem Buch einen reinen betriebswirtschaftlichen Ratgeber erwartet, wird enttäuscht werden. Herrn Jans gelingt es, die betriebswirtschaftlichen Grundlagen mit wichtigen persönlichen Aspekten und Fragestellungen zu verknüpfen. Dies führt zu einem durchweg interessant zu lesenden Werk.

Die zahlreichen betriebswirtschaftlichen Fachbegriffe, die viele schon häufig gehört, aber selten verstanden haben, werden für den Leser sehr einfach und verständlich, auch anhand von Praxisbeispielen, aufbereitet, sodass nach dem Lesen dieses Buches ein absolutes Grundverständnis für zahnärztliche Betriebswirtschaft besteht. Auch auf die Themen Versicherungen, Absicherungen und Familie wird eingegangen. Ein längeres Kapitel widmet der Autor den verschiedenen Darlehensformen, samt ihrer individuellen Vor- und Nachteile. Das Thema "Marketing" kommt ebenfalls nicht zu kurz.

Die beiden Beispielfälle, anhand derer das Buch aufgebaut wurde, sind extrem gut gewählt und sehr praxisnah. Herr Jans schafft es, durch die lockere Schreibweise einen Spannungsbogen zu kreieren, der dazu führt, dass sein Buch so kurzweilig ist.

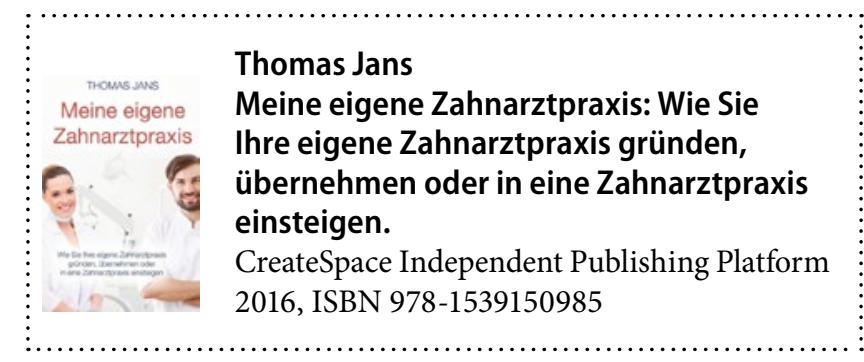

ANZEIGE

Hier steht eine Anzeige.

\section{Springer}

Mit dem Erwerb des Buches erhält der Leser Zugang zu viel nützlichem Bonusmaterial, welches online zugänglich ist. Am Ende eines jeden Kapitels gibt der Autor noch persönliche Praxistipps.

Fazit

"Meine eigene Zahnarztpraxis“ ist nicht nur ein Muss für Zahnmediziner, die den Schritt in die Selbstständigkeit planen oder unsicher in ihrer Entscheidung sind, sondern auch für schon selbstständige Zahnmediziner, die hiermit ihr betriebswirtschaftliches Wissen und ihren eingeschlagenen Weg überprüfen und ggf. korrigieren können.

Mir hätte dieses Buch bei meiner Selbstständigkeit viel Zeit und Mühe erspart. Doch auch im Nachhinein habe ich sehr von dem Inhalt profitiert. Zudem liest es sich nicht wie ein Verkaufsbuch für Praxisberater - es ist eindeutig zu erkennen, dass der Autor den Leser vor Fehlern bewahren möchte. „Meine eigene Zahnarztpraxis" von Thomas Jans kann von mir uneingeschränkt weiterempfohlen werden. 